\title{
CD36 Is Significantly Correlated with Adipophilin in Human Carotid Lesions and Inversely Correlated with Plasma ApoAI
}

\author{
Sophie Collot-Teixeira, ${ }^{1,2}$ Calypso Barbatis, ${ }^{3}$ Florence Bultelle, ${ }^{2}$ Michael Koutouzis, ${ }^{3}$ \\ Gerard Pasterkamp, ${ }^{4}$ Paul Fraser, ${ }^{1}$ Zenon S. Kyriakides, ${ }^{3}$ Robin N. Poston, ${ }^{1}$ Angelique Ristagno, ${ }^{2}$ \\ Lilian McGregor, ${ }^{5}$ Chantal M. Boulanger, ${ }^{6}$ Guy Leseche, ${ }^{7}$ and John L. McGregor ${ }^{1,2,6}$ \\ ${ }^{1}$ Cardiovascular Division, King's College London, University of London, Franklin-Wilkins Building, 150 Stamford Street, \\ London SE1 9NH, UK \\ ${ }^{2}$ Genomics and Atherothrombosis Laboratory, Thrombosis Research Institute, London SW3 6LR, UK \\ ${ }^{3}$ Department of Pathology, Helenic Red Cross Hospital, Athens, Greece \\ ${ }^{4}$ Laboratory of Experimental Cardiology, Utrecht University Hospital, Heidelberglaan 100, 3584 CX Utrecht, The Netherlands \\ ${ }^{5}$ EA 3470/INSERM IFR63, Faculty of Medicine RTH Laennec, Claude Bernard-Lyon 1 University, 69372 Lyon Cedex 08, France \\ ${ }^{6}$ INSERM U689, Centre de Recherche Cardiovasculaire, Hôpital Lariboisière, 75010 Paris, France \\ ${ }^{7}$ Vascular Surgery Department, Bichat Hospital, 75018 Paris, France
}

Correspondence should be addressed to Sophie Collot-Teixeira, sophie.collot@kcl.ac.uk

Received 8 April 2008; Revised 18 June 2008; Accepted 29 July 2008

Recommended by Mohamed Boutjdir

OxLDL uptake and cholesterol efflux inhibition in macrophages play a key role in atherosclerotic plaque formation, rupture, and thrombotic ischemia. This study investigates genes implicated in OxLDL uptake (CD36, SRA), cholesterol efflux inhibition (adipophilin, ADFP), and inflammatory recruitments of leukocytes (IL-8) in plaque lesion areas (PLAs) compared to nonplaque lesion areas (NPLAs) in human carotid endarterectomy specimens. Gene and protein expressions were assayed using quantitative PCR and quantitative immunohistochemistry. Pearson tests were used to investigate potential correlation between (a) different gene expressions and (b) gene expression and patient's plasma constituents. CD36, SRA, ADFP, and IL- 8 were shown to be significantly more expressed in PLA compared to NPLA. In PLA, a significant correlation was observed between CD36, SRA, ADFP, and IL-8 mRNA levels. Moreover, CD36 expression level was significantly inversely correlated to plasma marker ApoAI. The above investigated genes/proteins may play a key role in the maturation of atherosclerotic lesions.

Copyright ( $) 2008$ Sophie Collot-Teixeira et al. This is an open access article distributed under the Creative Commons Attribution License, which permits unrestricted use, distribution, and reproduction in any medium, provided the original work is properly cited.

\section{INTRODUCTION}

Macrophage uptake of oxidised low density lipoprotein (OxLDL) and the concomitant reduction in cholesterol efflux are thought to play a critical role in atherosclerotic plaque formation and subsequent rupture. A number of macrophage OxLDL receptors have been identified (e.g., CD36, SRBI, SRA) $[1,2]$ but two in particular, CD36 and SRA, have been shown to be responsible for up to $90 \%$ of OxLDL uptake and degradation by macrophages [3]. A double knockout mouse model of atherosclerosis $\left(\mathrm{ApoE}^{-/-}\right.$, $\mathrm{CD} 36^{-/-}$) has many fewer and smaller lesions compared to $\mathrm{ApoE}^{-/-}$animals that express CD36, even after long-term feeding with a western diet, $[4,5]$ which indicates that the CD36 scavenger receptor is implicated in the generation of advanced plaques. This is, however, at variance with the finding that similar double knockout mice have a comparable, or even increased, atherosclerotic lesions in the region of the aortic valve [6] as the $\mathrm{ApoE}^{-/-}$mice that have intact $\mathrm{CD} 36$, indicating that uptake and endocytosis of OxLDL seem to be independent of CD36 [6]. Different possibilities have been recently reviewed to explain such discrepancies $[7,8]$. Furthermore, results from $\mathrm{ApoE}^{-/-}$mice have to be interpreted cautiously, as the lesions are different from those observed in humans, often with none of the clinical end points, such as thrombotic ischaemia, resulting in myocardial infarction or stroke [9]. The present work has attempted to overcome this limitation by examining human carotid endarterectomy specimens. Indeed, very few studies have investigated the expression of CD36 in human vascular lesions [10]. 
Adipophilin (ADFP), a cellular indicator of the amount of stored lipids, is present in a very large number of tissues [11]. Increased expression of ADFP in THP-1 monocytic cell line has been observed to induce cholesterol storage and reduction of cholesterol efflux [12]. Moreover, its expression in macrophages has been correlated with the presence of red blood cells and cholesterol crystals in vascular lesions [13]. Finally, ADFP may play a role in the differentiation of macrophages into foam cells [14].

High density lipoproteins (HDLs) are implicated in the transport of cholesterol to the liver. It is now well established that there is an inverse relationship between the risk of developing cardiovascular diseases and the concentration of HDL. However, the protective effect of HDL is not fully understood. APOAI, the main apolipoprotein of HDL, plays a critical role in cholesterol efflux from macrophages and foam cells present in the vessel wall [15].

This study investigates genes/proteins implicated in OxLDL uptake (CD36, SRA), inhibition of cholesterol efflux (ADFP), and inflammatory recruitments of leukocytes (IL-8) in plaque lesion areas (PLAs) compared to nonplaque lesion areas (NPLAs) in human carotid endarterectomy samples. A substantial difference in mRNA and protein expressions between PLA and NPLA was observed for CD36, ADFP, SRA, and IL-8. In addition, a significant correlation was obtained between CD36 expression and ADFP, SRA and IL8 in PLA. Moreover, a significant inverse correlation was observed between CD36 gene expression level in PLA and plasma marker ApoAI.

\section{MATERIALS AND METHODS}

\subsection{Human tissue samples}

Ninety patients, subjected to endarterectomy (carotid stenosis $>70 \%$ ) at 3 different hospitals (Red Cross Hospital in Athens (Greece), University Medical Centre in Utrecht (The Netherlands), and Bichat hospital in Paris (France)), were enrolled in this study. After removal, endarterectomy samples were immediately rinsed in RNAse-free sterile PBS, submerged in RNALater (Ambion, Tex, USA), and stored at $-80^{\circ} \mathrm{C}$. Ethical committees approved the study, and patients were fully informed and gave their written consent prior to enrolment. Information concerning the patients is summarised in Table 1.

\subsection{Endarterectomy handling}

The lesions were classified according to the American Heart Association (AHA) criteria [16] by expert pathologists (Table 1). A transverse (perpendicular to the lumen) section of the internal carotid artery of 5-10 $\mu \mathrm{m}$ thickness was taken in the plaque lesion area (PLA) above the bifurcation point (Figure 1). When available, a macroscopically nonplaque lesion area (NPLA) adjacent to the atheromatous area, removed as a part of the normal surgical procedure, was used as an internal control. The PLA and NPLA sections were split horizontally giving 2 identical pieces of the same area being used, respectively, for immunohistochemistry and for
TABle 1: Patient phenotypes.

\begin{tabular}{|c|c|}
\hline & $n=90$ \\
\hline Age & $66.5 \pm 8.7$ \\
\hline Male/Female & $76 \% / 24 \%$ \\
\hline Smokers & $64 \%$ \\
\hline Symptoms & $56 \%$ \\
\hline Diabetes mellitus & $26.8 \%$ \\
\hline Hypertension & $84 \%$ \\
\hline Total cholesterol (mM) & $4.8 \pm 0.9$ \\
\hline $\mathrm{HDl}(\mathrm{mM})$ & $1.01 \pm 0.19$ \\
\hline $\mathrm{LDL}(\mathrm{mM})$ & $3.1 \pm 0.8$ \\
\hline Triglycerides (mM) & $3.9 \pm 2.3$ \\
\hline Hematocrit (\%) & $40 \pm 4.7$ \\
\hline White blood cells $\left(* 10^{9} / \mathrm{L}\right)$ & $7.3 \pm 1.4$ \\
\hline Platelets $\left(* 10^{9} / \mathrm{L}\right)$ & $218(115-360)$ \\
\hline Glucose (mg/dL) & $93 \pm 27.6$ \\
\hline Creatinine (mg/dL) & 0.9 \\
\hline beta-blokers & $32.8 \%$ \\
\hline calcium antagonists & $30.1 \%$ \\
\hline ACE inhibitors & $42.5 \%$ \\
\hline Diuretics & $29 \%$ \\
\hline $\mathrm{ARB}$ & $26 \%$ \\
\hline Statins & $49 \%$ \\
\hline Aspirin & $47.9 \%$ \\
\hline Clopidogrel & $46.2 \%$ \\
\hline Insulin & $3 \%$ \\
\hline Diabetes mellitus pills & $21.9 \%$ \\
\hline ApoAI (mg/dL) & $115 \pm 18$ \\
\hline $\operatorname{ApoB}(\mathrm{mg} / \mathrm{dL})$ & $92 \pm 23$ \\
\hline $\operatorname{Lp}(\mathrm{a})$ & $20 \pm 48$ \\
\hline \multirow[t]{4}{*}{ Histological grade (AHA) } & $65.7 \%$ grade 6 \\
\hline & $28.6 \%$ grade 5 \\
\hline & 4.3 grade 4 \\
\hline & $1.4 \%$ grade 3 \\
\hline
\end{tabular}

quantitative PCR (Q-PCR) (Figure 1). Fibroatheroma (type $\mathrm{V}$ ) or complicated (type VI) lesions, as described in the AHA criteria constituted the investigated PLA. Both pieces were washed twice in cold RNAse-free sterile PBS to remove the RNALater and either included into OCT or immersed into RLT lysis buffer (Qiagen, Calif, USA) to protect the RNA and stored at $-80^{\circ} \mathrm{C}$ until further use. For all the samples included into OCT, tissue sections were cut at $8 \mu \mathrm{m}$ thickness, dried, and fixed in acetone. Plaque morphology was also evaluated by staining the tissues with hematoxylin and eosin.

\subsection{THP-1 cells culturing and stimulation}

Human monocytic leukaemia cell line (THP-1/European collection of cell cultures-ECACC no. 88081201) was cultured in RPMI 1640 (Sigma, Mo, USA) supplemented with $10 \%$ heat-inactivated foetal calf serum (FCS) (Sigma), $1 \%$ glutamine (Sigma), $100 \mu \mathrm{g} / \mathrm{mL}$ streptomycin (Sigma) in a humid incubator with $5 \% \mathrm{CO}_{2}$ at $37^{\circ} \mathrm{C}$. THP-1 cells, 


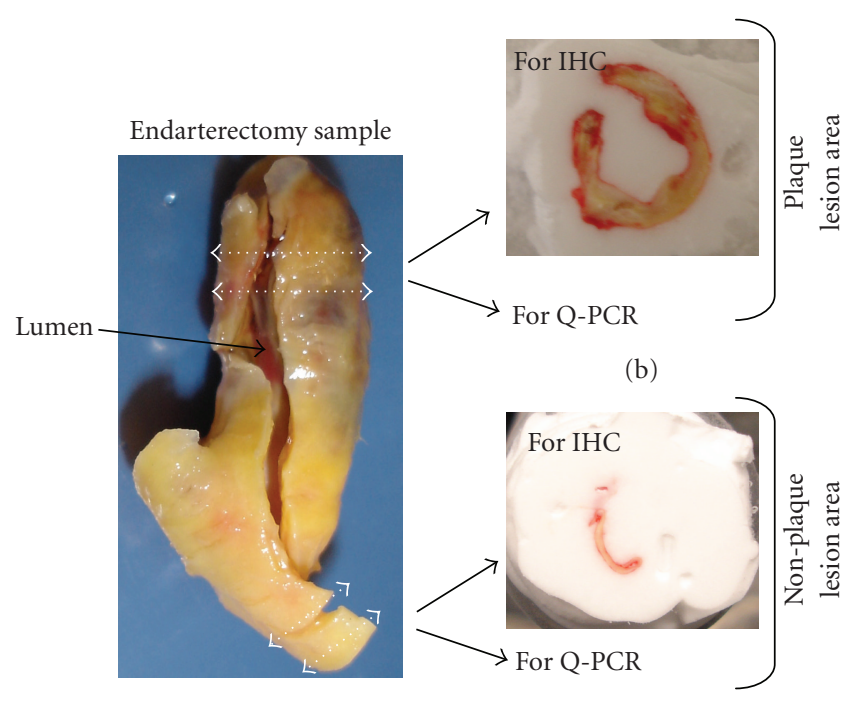

(a)

(c)

FIGURE 1: (a) Typical macroscopic pictures of a carotid endarterectomy specimen. (b) A transverse (perpendicular to the lumen) section of the internal carotid artery was taken in the plaque lesion area (PLA) above the bifurcation point. (c) A macroscopically nonplaque area (NPLA) was used as an internal control. The PLA and the NPLA sections were split horizontally giving 2 identical pieces that were used, respectively, for immunohistochemistry and for quantitative PCR.

cultured in 6-well plates at a density $1.2 \times 10^{6}$ cells/well, were first treated with $0.2 \mu \mathrm{M}$ phorbol 12 -myristate 13 acetate (PMA, Sigma) during 24 hours in order to induce the differentiation of monocytes into macrophages, then washed with sterile PBS and stimulated with $100 \mu \mathrm{g} / \mathrm{mL}$ oxidised low-density lipoproteins (OxLDL, Intracell, USA) during 24 hours. Cell viability was evaluated using Trypan blue counting to be more than $95 \%$ for each assay. Stimulation assays were performed in duplicate and repeated 3 times.

\subsection{RNA extraction}

THP-1 cells: total RNA extractions were performed using the RNeasy Mini Kit (Qiagen) following manufacturer's instructions. RNA quality and quantity were monitored using a Nanodrop spectrophotometer (Nanodrop Technologies, Del, USA) and an RNA 6000 NanoLabChip kit on a Bioanalyser (Agilent Technologies, Calif, USA).

Tissue samples: 10 to $30 \mathrm{mg}$ of tissue were used for RNA extraction depending on the tissue size available. Tissues were first cut into very small pieces and homogenised with $350 \mu \mathrm{L}$ of RLT lysis buffer (Qiagen) using a Polytron $(2 \times 30 \mathrm{~s}$ on ice $)$. After 10 minutes at $55^{\circ} \mathrm{C}$ proteinase $\mathrm{K}$ step $\left(0.22 \mathrm{~g} / \mathrm{L}^{-1}\right)$, the tissue lysate was centrifuged 3 minutes at $10.000 \mathrm{~g}$ and RNA extraction was performed on the supernatant following manufacturer's instructions using RNeasy Mini Kit (Qiagen). RNA quality and quantity were monitored using RNA 6000 PicoLabChip/NanoLabChip kit on a Bioanalyser (Agilent) and using the Nanodrop spectrophotometer (Nanodrop Technologies). Total RNA extracted from highly calcified lesions was poor and these were removed from the cohort.

\subsection{Retrotranscription (RT) and real-time quantitative PCR (Q-PCR)}

Only total RNA extracts presenting good integrity on Bioanalyser profiles were retrotranscribed ( $1 \mu \mathrm{g}$ for THP1 cells or $200 \mathrm{ng}$ for tissue samples) with superscript III reverse transcriptase (Invitrogen) following manufacturer's instructions.

Q-PCR assays were carried out using the $7900 \mathrm{HT}$ Sequence Detector System (Applied Biosystems/Ambion, $(\mathrm{AB}))$. Five microlitres of 10X RT product were amplified using 1X of Universal PCR Master Mix (AB), 1X assayon-demand (containing specific primers and labelled probe, $\mathrm{AB}$ ) in a final volume of $25 \mu \mathrm{L}$. Q-PCR amplification was performed in duplicate. 18S rRNA (AB) amplification was used for normalisation. Each gene was assayed individually.

\subsection{Immunohistochemistry and quantitative image analysis}

Serial sections of endarterectomy samples (NPLA and PLA) were first immunostained with an anti-CD68 (1/500, Dako, Glostrup, Denmark), an antialpha actin (1/50, Dako), and an anti-CD31 (1/20, Dako) in order to localise macrophages, smooth muscle cells (SMCs), and endothelial cells, respectively. Then sections were incubated with either an antiCD36 monoclonal antibody (1/25, BioCytex, Marseille, France), an anti-SRA (1/100, R\&D Systems, Minn, USA), an anti-ADFP (1/200, Chemicon, Millipore, Billerica, USA), or the corresponding IgG isotype. Staining was visualised using the Envision kit (Dako). Slides were then counterstained with hematoxylin and mounted in DPX (Sigma). Staining of CD36, SRA, and ADFP was quantitatively analysed by the use of a colour image analyser (Sight System, Newbury, Berks, UK; Software by Foster-Finley Associates, Newcastle, UK) detecting the peroxidase reaction product by measurement of pixels in terms of hue, saturation, and intensity values. By this method, the percentage of the stained tissue was measured. For each tissue, the entire section was analysed and the intensity staining obtained was normalised via the surface of the tissue analysed $[17,18]$.

\subsection{Western-blot}

Proteins were extracted using 5\%-tritonX-100 lysis buffer. Twenty micrograms of total proteins were subjected to SDS-polyacrylamide gel electrophoresis and transferred to nitrocellulose membranes. The membranes were incubated with an anti-CD36 monoclonal antibody (Clone 10/5) (BioCytex), or with an anti-ADFP polyclonal antibody (AB9102) (Chemicon) at dilutions $1 / 100$ and 1/2000, respectively. Detection was performed using ECL western blotting detection reagents (Amersham, Buckinghamshire, UK) and exposure to Kodak Biomax XAR films (Sigma). Signals were analysed and compared using GelDoc software (BioRad Laboratories, Calif, USA). 


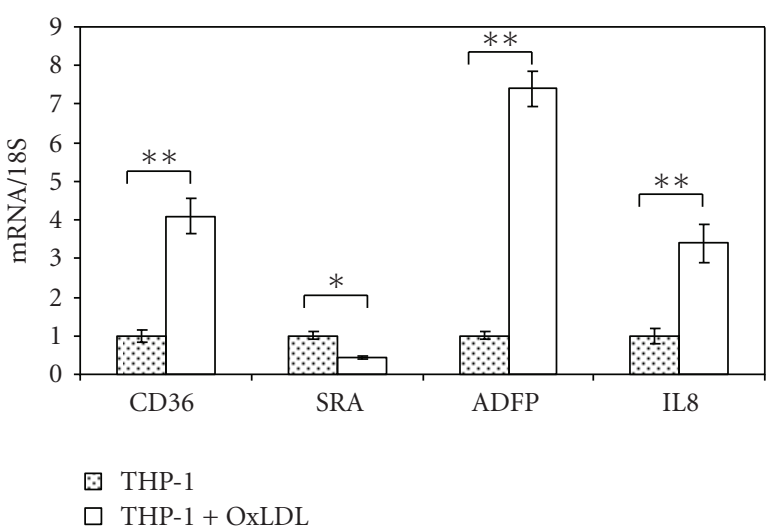

(a)

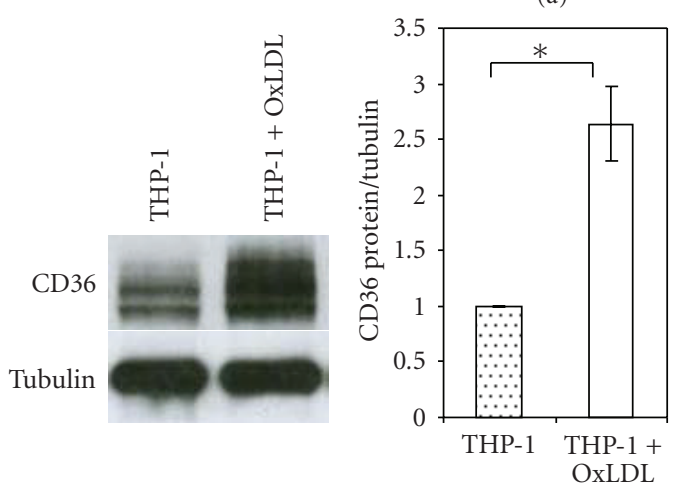

(b)

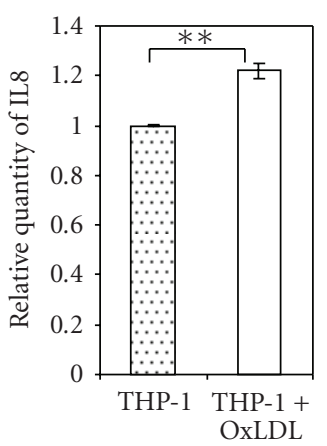

(d)

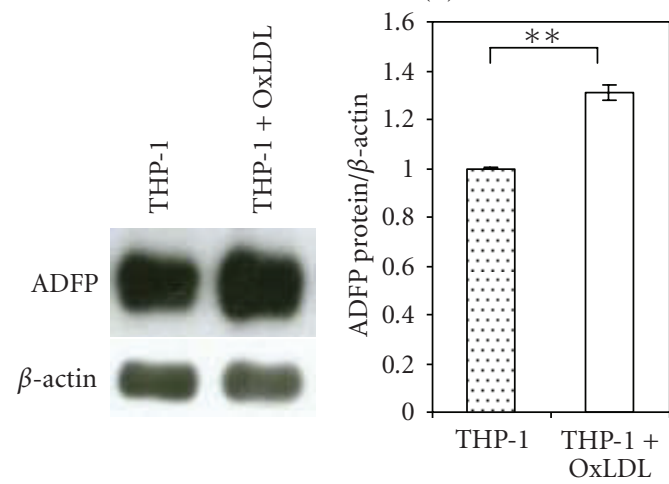

(c)

FIGURe 2: Comparison of gene/protein expression patterns between OxLDL-treated (100 $\mu \mathrm{g} / \mathrm{mL}$, for 24 hours $)$ and PBS-treated PMAdifferentiated THP-1 cells. (a) Real-time quantitative PCR showing gene expression levels for CD36, SRA, adipophilin (ADFP), and IL-8. (b), (c) Western-blot analysis of, respectively, CD36 and ADFP protein expression levels. (d) IL-8 protein levels in THP-1 culture supernatants assayed by ELISA. Treated and untreated cells are, respectively, represented by plain and dotted bars. Bars represent the means \pm SE for 3 different experiments performed in duplicate. Wilcoxon matched pairs test was used to determine significance. ${ }^{* *} P \leq .01, * P \leq .05$.

\subsection{ELISA}

IL-8 levels were measured in cell supernatants in duplicate using an IL-8 ELISA Kit (Quantikine from R\&D Systems) accordingto the supplier's instructions.

\subsection{Statistical analysis}

Statistical analyses were performed using Wilcoxon matched pairs tests (ranks test) and Mann-Whitney tests (Statistica software, Statsoft). Online software (http://www.fon.hum. uva.nl/Service/Statistics/Correlation_coefficient.html) was used to conduct Pearson's correlation tests. In all cases, a value of $P \leq .05$ was regarded as significant.

\section{RESULTS}

\subsection{OxLDL stimulation in PMA-differentiated THP-1 macrophages}

An in vitro approach was first used to investigate the gene and protein expressions, under our laboratory conditions, of 4 OxLDL-targeted genes (CD36, SRA, ADFP, IL-8) prior to starting the work on endarterectomy tissues. Twenty four hours of OxLDL treatment $(100 \mu \mathrm{g} / \mathrm{mL})$ of PMAdifferentiated THP-1 macrophages, compared to cells incubated with PBS only, induced a significant increase of CD36 $(4.1 \pm 1.1$-fold $)$, IL-8 $(3.4 \pm 0.9$-fold $)$, and ADFP $(7.4 \pm 1.4$ fold) gene expressions, $P<.05$ (Figure 2(a)). In contrast, a decrease of SRA gene expression $(0.5 \pm 0.1$-fold $)$ was observed in these cells after OxLDL treatment $(100 \mu \mathrm{g} / \mathrm{mL}) P<.05$. Western-blot analysis showed that CD36 and ADFP protein levels were also increased respectively by $132 \%( \pm 30 \%)(P=$ $.05)$ and by $31 \%( \pm 3 \%, P=.01)$ after the OxLDL treatment (Figures 2(b) and 2(c)). IL-8 increased by $22 \%( \pm 1.1 \%, P=$ $.005)$ in cell supernatant using ELISA assays (Figure $2(\mathrm{~d})$ ).

\subsection{Comparison of gene and protein expressions in PLA versus NPLA}

Gene and protein expressions (CD36, SRA, ADFP, and IL-8) at sites of PLA were compared to NPLA in endarterectomy samples. CD36 (6.80 versus 5.41, $P=.0045)$, ADFP (6.85 versus $5.78, P=.0017)$, SRA (6.93 versus $6.27, P=.23$ ), and IL-8 (5.98 versus 4.93, $P=.0065)$ genes were shown to be significantly more expressed in PLA compared to NPLA in the 15 patient's samples investigated (Figure 3(a)). Furthermore, PLA showed significantly higher CD36 

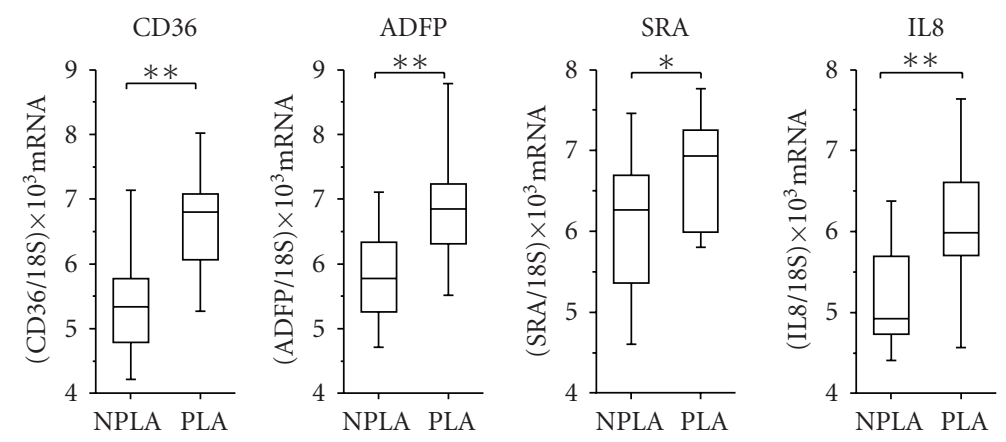

(a)
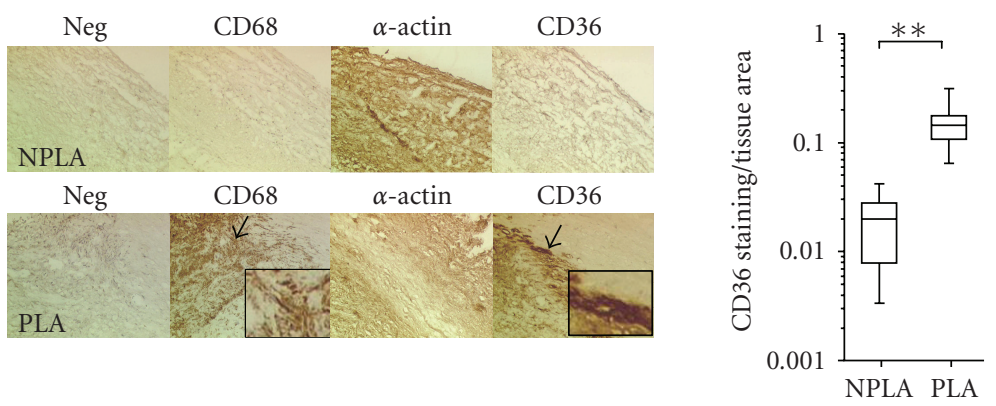

(b)
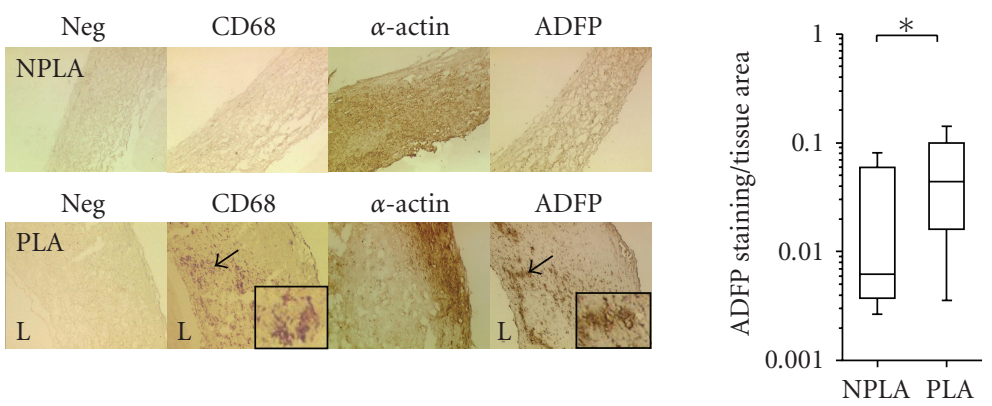

(c)
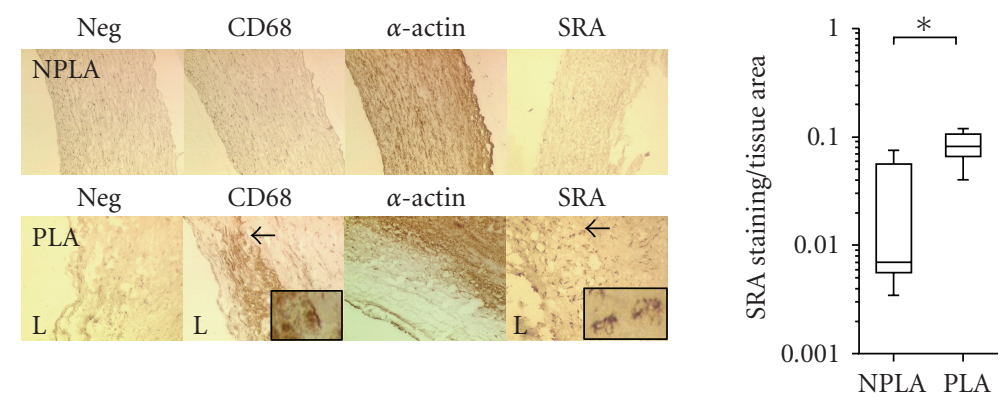

(d)

FIGURE 3: Gene and protein expression levels in carotid endarterectomy PLAs (plaque lesion areas) and NPLAs (nonplaque lesion areas). (a) Real-time quantitative PCR showing gene expression levels for CD36, adipophilin (ADFP), SRA, and IL-8 in carotid endarterectomy PLA compared to NPLA. (b) Staining of negative isotype, CD68, $\alpha$-actin, and CD36 proteins in PLA and NPLA and quantitative immunohistochemistry for CD36 protein expression in PLA versus NPLA. (c) Staining of negative isotype, CD68, $\alpha$-actin, and ADFP proteins in PLA and NPLA and quantitative immunohistochemistry for ADFP protein expression in PLA versus NPLA. (d) Staining of negative isotype, CD68, $\alpha$-actin, and SRA proteins in PLA and NPLA and quantitative immunohistochemistry for SRA protein expression in PLA versus NPLA. Wilcoxon matched pairs test was used to determine significance. ${ }^{* *} P \leq .001,{ }^{*} P \leq .05$; L: lumen; magnification: $10 \mathrm{X}$. 


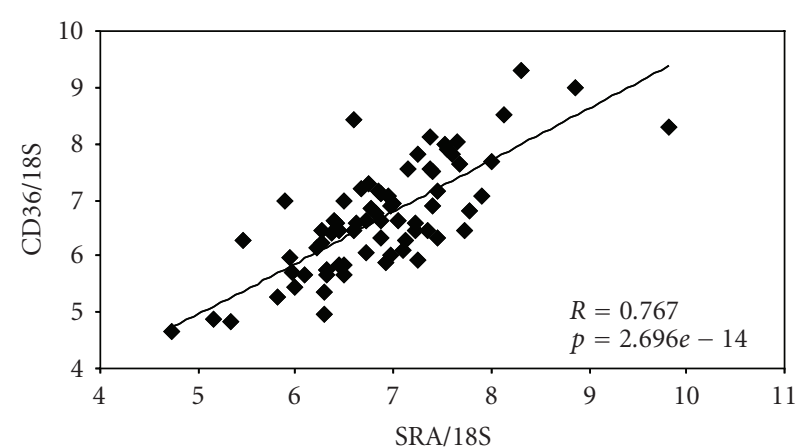

(a)

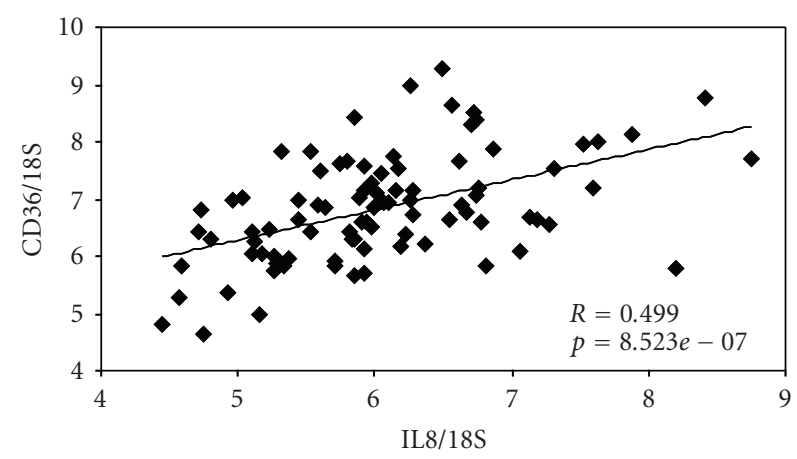

(c)

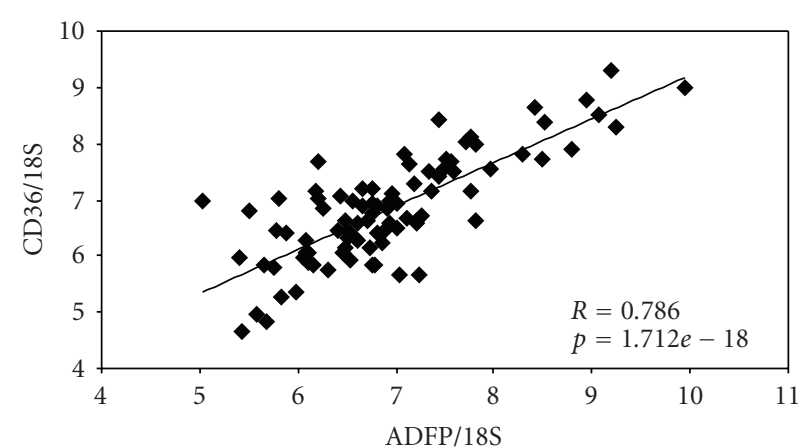

(b)

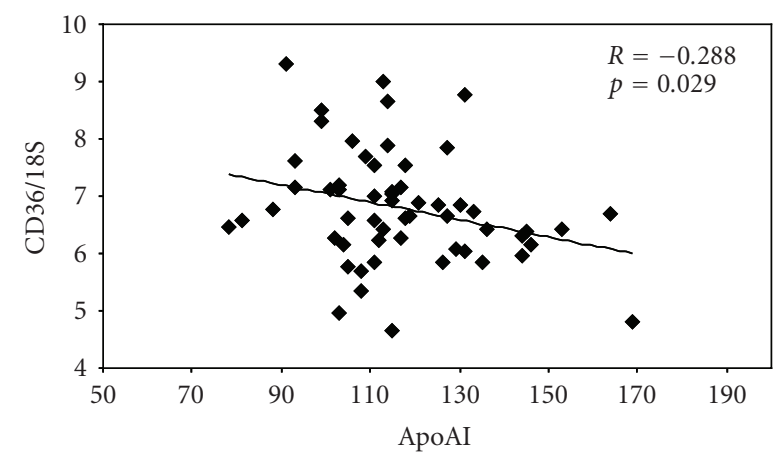

(d)

FIGURE 4: Correlation of CD36 with (a) SRA $(n=72)$, (b) adipophilin $(n=88)$, or (c) IL-8 $(n=88)$ gene expression in plaque lesion area (PLA). (d) Correlation of CD36 gene expression in plaque lesion areas (PLAs) with plasma marker ApoAI level in 57 patients. Pearson's correlation statistical test was used.

(0.15 versus $0.019, P=.017)$, ADFP (0.051 versus 0.026, $P=.049)$, and SRA (0.081 versus $0.027, P=.027)$ protein levels compared to NPLA using quantitative immunostaining techniques in the 8 patient's samples investigated (Figures 3(b), 3(c), and 3(d)).

\subsection{Correlation of CD36 with SRA or ADFP in carotid endarterectomy specimens}

In PLA samples, a significant correlation was observed between CD36 and SRA mRNA expression levels $(n=72$, $R=.767, P<.01)$. Interestingly, a significant correlation was also observed between CD36 and ADFP $(n=88, R=$ .786, $P<.01)$ and between CD36 and IL-8 $(n=88, R=$ $.499, P<.01)$ mRNA expression levels (Figure 4$)$.

\subsection{Correlation between CD36 with ApoAl and HDL}

A significant inverse correlation was observed between CD36 gene expression level in PLA samples $(n=57)$ and plasma marker ApoAI $(R=.288, P=.029)$ (Figure $4(d))$. Furthermore, an inverse correlation was also found between CD36 gene expression and HDL plasma level, however it was not statistically significant $(R=.207, P=.1)$. No correlation was observed between CD36 and total cholesterol, LDL, triglycerides, plasma ApoB, medication, smoking status or gender (data not shown).

\section{DISCUSSION}

In this study, a substantial difference in mRNA and protein expressions was observed for CD36 and molecules implicated in lipid metabolism (ADFP, SRA) and inflammation (IL-8) between plaque lesion area (PLA) and nonplaque lesion area (NPLA) in human carotid endarterectomies. Moreover, results obtained for an in vitro macrophage model (PMA-treated THP-1 cells) stimulated by OxLDL show a trend, with the exception of SRA, that is similar to those obtained in PLA. In addition, a significant correlation was obtained between CD36 expression and ADFP, SRA, and IL8 in PLA. Furthermore, a significant inverse correlation was observed between CD36 gene expression level in PLA and plasma marker ApoAI.

CD36, ADFP, and SRA are strongly correlated in this study in PLA and are potentially proatherogenic proteins. Indeed, resident vascular macrophages have scavenger receptors CD36 and SRA that play a key role in the uptake of OxLDL. Moreover, ADFP in these very same macrophages is involved in lipid accumulation by inhibiting efflux [12]. It has also been described that the ABCA1 transporter protein expression, involved in the cholesterol transport from cells to apolipoproteins in plasma, was significantly reduced in carotid lesions compared with controls tissues [19]. The uptake of OxLDL upregulates scavenger receptors and the adipophilin expression and ensures the formation of foam cells and the perpetuation of lesions. An inflammatory 


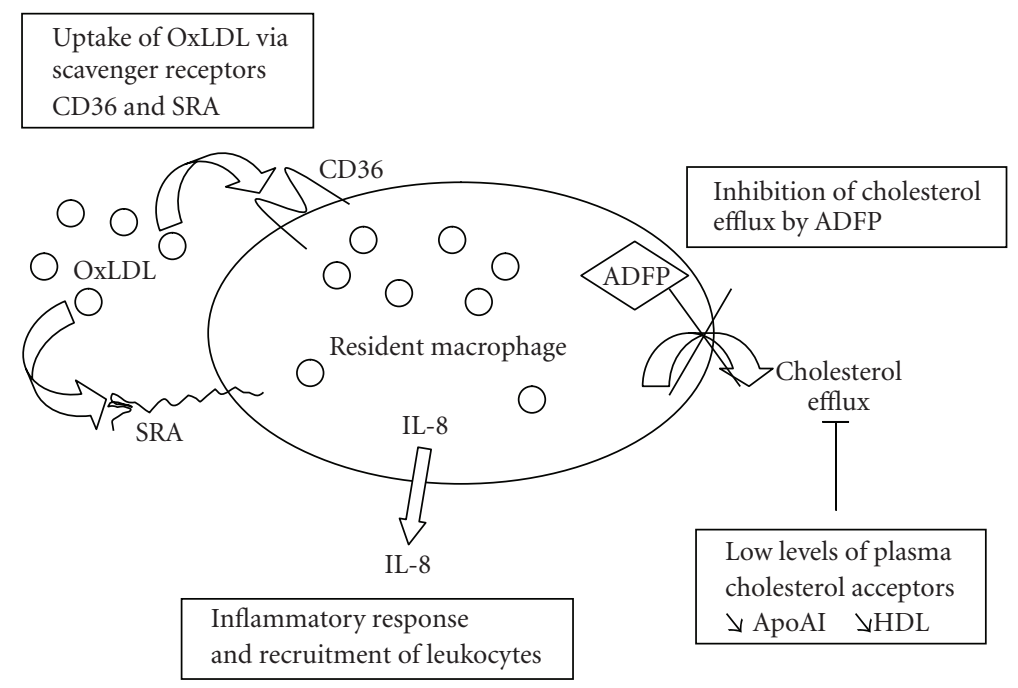

FIGURE 5: Hypothetical representation of OxLDL influx and inhibition of cholesterol efflux in carotid resident macrophages. In the presence of high expression of CD36 in plaque lesion areas, the observed strong correlation between CD36, SRA, and ADFP suggests that carotid lesion resident macrophages are subjected on the one hand to greatly enhanced OxLDL uptake by CD36 and SRA and on the other to inhibition of cholesterol efflux by ADFP. The cholesterol efflux from resident macrophages is probably even further reduced as indicated by the inverse correlation of CD36 expression with plasma cholesterol acceptor ApoAI.

component (IL-8) that is correlated to CD36 in PLA is also observed. This brings a further dimension to the capacity of resident macrophages to attract further monocytes to the sites of inflammation through the release of IL-8. An additional interesting finding in this study is that the plasma acceptor ApoAI, a constituent of HDL, needed for transportation of cholesterol from macrophages is significantly inversely correlated to CD36 gene expression level in PLA. Macrophages do not synthesise ApoAI and need to have it readily available in plasma as a cholesterol acceptor. In the absence of ApoAI, cholesterol efflux is greatly reduced. HDL levels were also inversely correlated to CD36 gene expression but this correlation did not reach statistical significance.

CD36 in this study is statistically more highly expressed in PLA (grade V and VI lesions, AHA classifications) [16] compared to NPLA regions. Cells expressing CD36 are mostly macrophages as indicated by CD68 labelling. CD36 in murine $\mathrm{ApoE}^{-/-}$macrophages, compared to $\mathrm{ApoE}^{-/-}$ $\mathrm{CD} 36^{-1-}$, has been shown in several studies to be linked to the generation of atherosclerotic lesions. However, a certain caution needs to be taken in trying to understand human atherosclerotic lesions compared to those generated in $\mathrm{ApoE}^{-/-}$mice. Indeed, in the $\mathrm{ApoE}^{-/-}$murine model of atherosclerosis there is an absence of myocardial infarction or stroke that are the results of clinical complications of atherosclerosis in patients. The intense CD36 labelling obtained in this study for PLA compared to NPLA suggests that this scavenger receptor is actively involved in the perpetuation of lesions in human vessels.

The PMA-differentiated THP-1 incubated with OxLDL showed a similar upregulation of CD36, ADFP, and IL-8 gene and protein expressions compared to those obtained in PLA versus NPLA. In contrast, SRA which is enhanced in PLA versus NPLA was shown to be decreased by OxLDL treatment in PMA-differentiated THP-1. It is conceivable that SRA and CD36 might be regulated differentially and therefore could play different roles in the formation of foam cells in atherosclerotic lesions. Indeed, CD36 and SRA are differently expressed in lesion area present in post-mortem descending human thoracic aorta [20]. Alternatively, a monocytic cell line such as THP-1 cells may differ in its response from normal monocytes/macrophages.

A certain caution needs to be taken in interpreting the above data. However, an interesting working hypothesis would rely on carotid resident macrophages to take up OxLDL via upregulated CD36 and SRA and inhibit cholesterol efflux as a result of the high expression of ADFP and low levels of plasma cholesterol acceptor ApoAI. Moreover, IL-8 would be implicated in monocyte recruitment to the sites of inflammation (Figure 5).

\section{ACKNOWLEDGMENTS}

The authors would like to warmly thank Dr. Brigitte McGregor for her assistance with the immunohistochemistry. S. Collot-Teixeira and J. L. McGregor are supported by a British Heart Foundation Grant (PG/05/090) at the Cardiovascular Division, King's College London, UK. J. L. McGregor is supported by a grant from INSERM Unit 689. In the early part of this work, S. Collot-Teixeira and J. L. McGregor were supported at the Thrombosis Research Institute Genomics and Atherothrombosis Laboratory by a Garfield Weston Foundation Grant.

\section{REFERENCES}

[1] T. Doi, K.-I. Higashino, Y. Kurihara, et al., "Charged collagen structure mediates the recognition of negatively charged 
macromolecules by macrophage scavenger receptors," The Journal of Biological Chemistry, vol. 268, no. 3, pp. 2126-2133, 1993.

[2] T. Mazzone, "Scavenger receptors in atherosclerosis: new answers, new questions," Arteriosclerosis, Thrombosis, and Vascular Biology, vol. 20, no. 12, pp. 2506-2508, 2000.

[3] V. V. Kunjathoor, M. Febbraio, E. A. Podrez, et al., "Scavenger receptors class A-I/II and CD36 are the principal receptors responsible for the uptake of modified low density lipoprotein leading to lipid loading in macrophages," The Journal of Biological Chemistry, vol. 277, no. 51, pp. 49982-49988, 2002.

[4] M. Febbraio, E. A. Podrez, J. D. Smith, et al., "Targeted disruption of the class B scavenger receptor CD36 protects against atherosclerotic lesion development in mice," The Journal of Clinical Investigation, vol. 105, no. 8, pp. 1049-1056, 2000.

[5] E. Guy, S. Kuchibhotla, R. Silverstein, and M. Febbraio, "Continued inhibition of atherosclerotic lesion development in long term Western diet fed $\mathrm{CD} 36^{\circ} / \mathrm{apoE}^{\circ}$ mice," Atherosclerosis, vol. 192, no. 1, pp. 123-130, 2007.

[6] K. J. Moore, V. V. Kunjathoor, S. L. Koehn, et al., "Loss of receptor-mediated lipid uptake via scavenger receptor A or CD36 pathways does not ameliorate atherosclerosis in hyperlipidemic mice," The Journal of Clinical Investigation, vol. 115, no. 8, pp. 2192-2201, 2005.

[7] S. Collot-Teixeira, J. Martin, C. McDermott-Roe, R. Poston, and J. L. McGregor, "CD36 and macrophages in atherosclerosis," Cardiovascular Research, vol. 75, no. 3, pp. 468-477, 2007.

[8] M. Febbraio and R. L. Silverstein, "CD36: implications in cardiovascular disease," The International Journal of Biochemistry \& Cell Biology, vol. 39, no. 11, pp. 2012-2030, 2007.

[9] S. M. Schwartz, Z. S. Galis, M. E. Rosenfeld, and E. Falk, "Plaque rupture in humans and mice," Arteriosclerosis, Thrombosis, and Vascular Biology, vol. 27, no. 4, pp. 705-713, 2007.

[10] Y. Nakagawa-Toyama, S. Yamashita, J.-I. Miyagawa, et al., "Localization of CD36 and scavenger receptor class A in human coronary arteries - a possible difference in the contribution of both receptors to plaque formation," Atherosclerosis, vol. 156, no. 2, pp. 297-305, 2001.

[11] C. Londos, D. L. Brasaemle, C. J. Schultz, J. P. Segrest, and A. R. Kimmel, "Perilipins, ADRP, and other proteins that associate with intracellular neutral lipid droplets in animal cells," Seminars in Cell \& Developmental Biology, vol. 10, no. 1, pp. 51-58, 1999.

[12] G. Larigauderie, C. Furman, M. Jaye, et al., "Adipophilin enhances lipid accumulation and prevents lipid efflux from THP-1 macrophages: potential role in atherogenesis," Arteriosclerosis, Thrombosis, and Vascular Biology, vol. 24, no. 3, pp. 504-510, 2004.

[13] K. Nuotio, P. M. Isoviita, J. Saksi, et al., "Adipophilin expression is increased in symptomatic carotid atherosclerosis: correlation with red blood cells and cholesterol crystals," Stroke, vol. 38, no. 6, pp. 1791-1798, 2007.

[14] X. Wang, T. J. Reape, X. Li, et al., "Induced expression of adipophilin mRNA in human macrophages stimulated with oxidized low-density lipoprotein and in atherosclerotic lesions,” FEBS Letters, vol. 462, no. 1-2, pp. 145-150, 1999.

[15] P. J. Barter and K.-A. Rye, "The rationale for using apoA-I as a clinical marker of cardiovascular risk," Journal of Internal Medicine, vol. 259, no. 5, pp. 447-454, 2006.

[16] H. C. Stary, A. B. Chandler, R. E. Dinsmore, et al., "A definition of advanced types of atherosclerotic lesions and a histological classification of atherosclerosis. A report from the Committee on Vascular Lesions of the Council on Arteriosclerosis,
American Heart Association," Circulation, vol. 92, no. 5, pp. 1355-1374, 1995.

[17] R. R. Johnson-Tidey, J. L. McGregor, P. R. Taylor, and R. N. Poston, "Increase in the adhesion molecule P-selectin in endothelium overlying atherosclerotic plaques. Coexpression with intercellular adhesion molecule-1," The American Journal of Pathology, vol. 144, no. 5, pp. 952-961, 1994.

[18] R. N. Poston, D. O. Haskard, J. R. Coucher, N. P. Gall, and R. R. Johnson-Tidey, "Expression of intercellular adhesion molecule-1 in atherosclerotic plaques," The American Journal of Pathology, vol. 140, no. 3, pp. 665-673, 1992.

[19] C. Albrecht, S. Soumian, J. S. Amey, et al., "ABCA1 expression in carotid atherosclerotic plaques," Stroke, vol. 35, no. 12, pp. 2801-2806, 2004.

[20] A. Nakata, Y. Nakagawa, M. Nishida, et al., "CD36, a novel receptor for oxidized low-density lipoproteins, is highly expressed on lipid-laden macrophages in human atherosclerotic aorta," Arteriosclerosis, Thrombosis, and Vascular Biology, vol. 19, no. 5, pp. 1333-1339, 1999. 

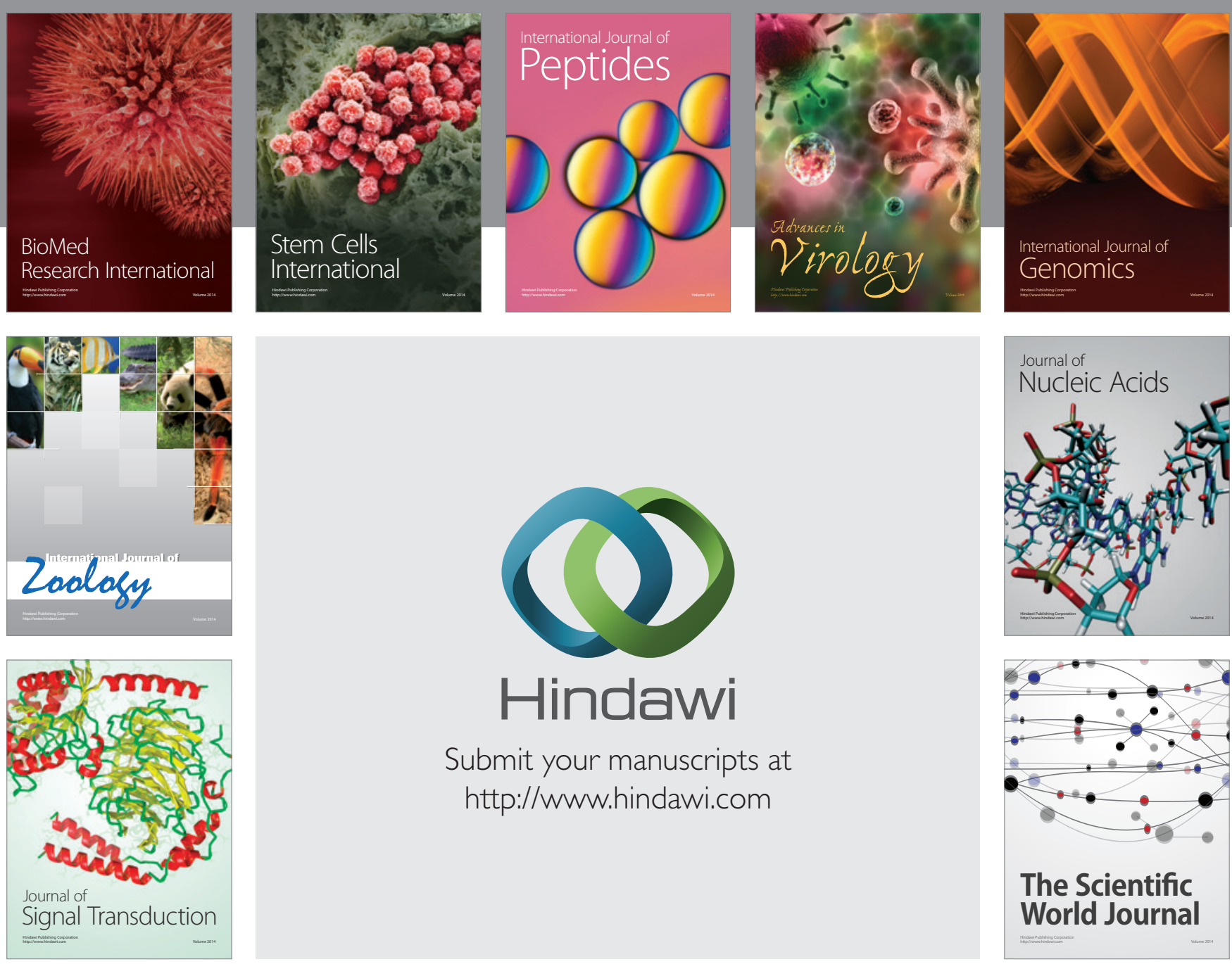

Submit your manuscripts at

http://www.hindawi.com
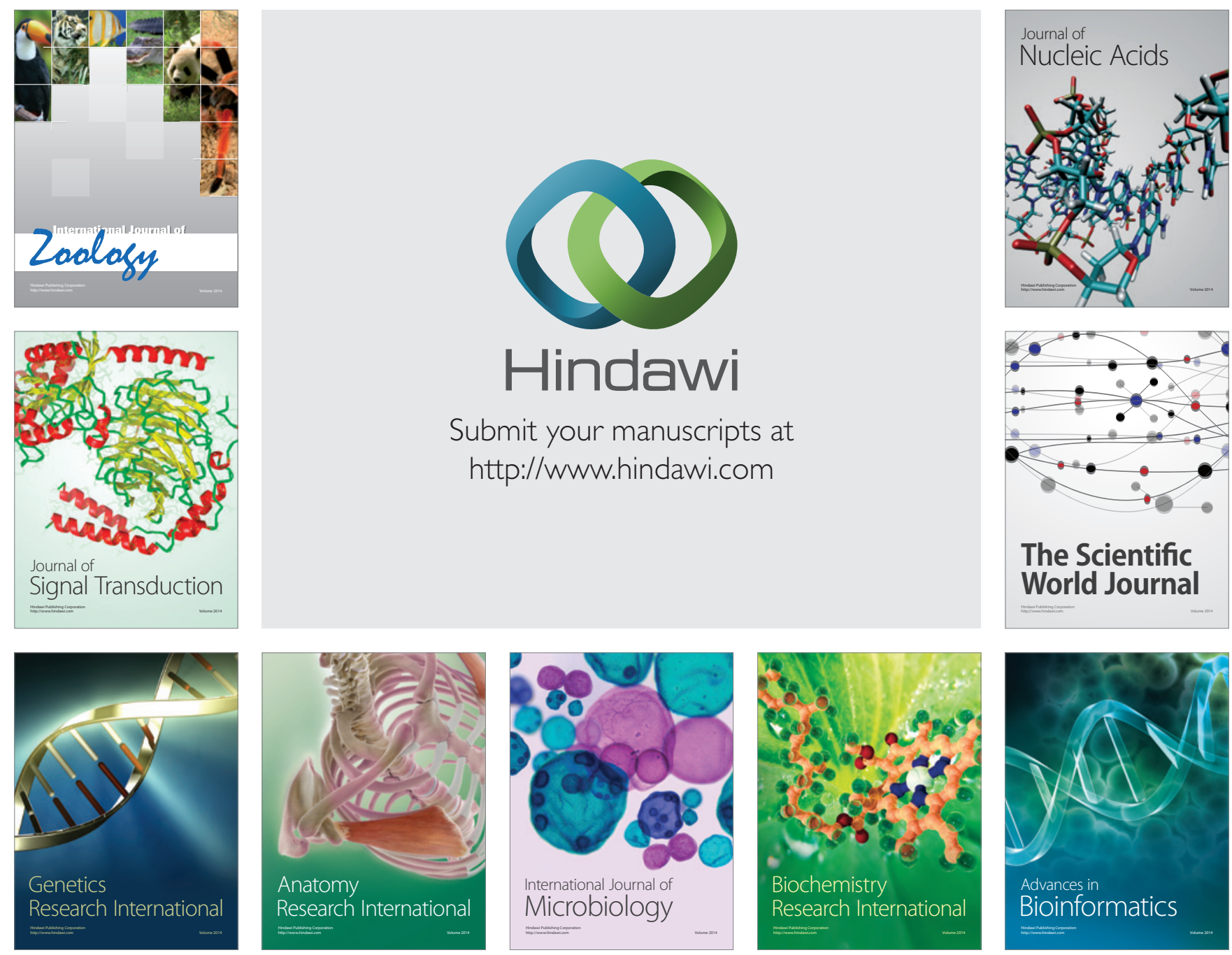

The Scientific World Journal
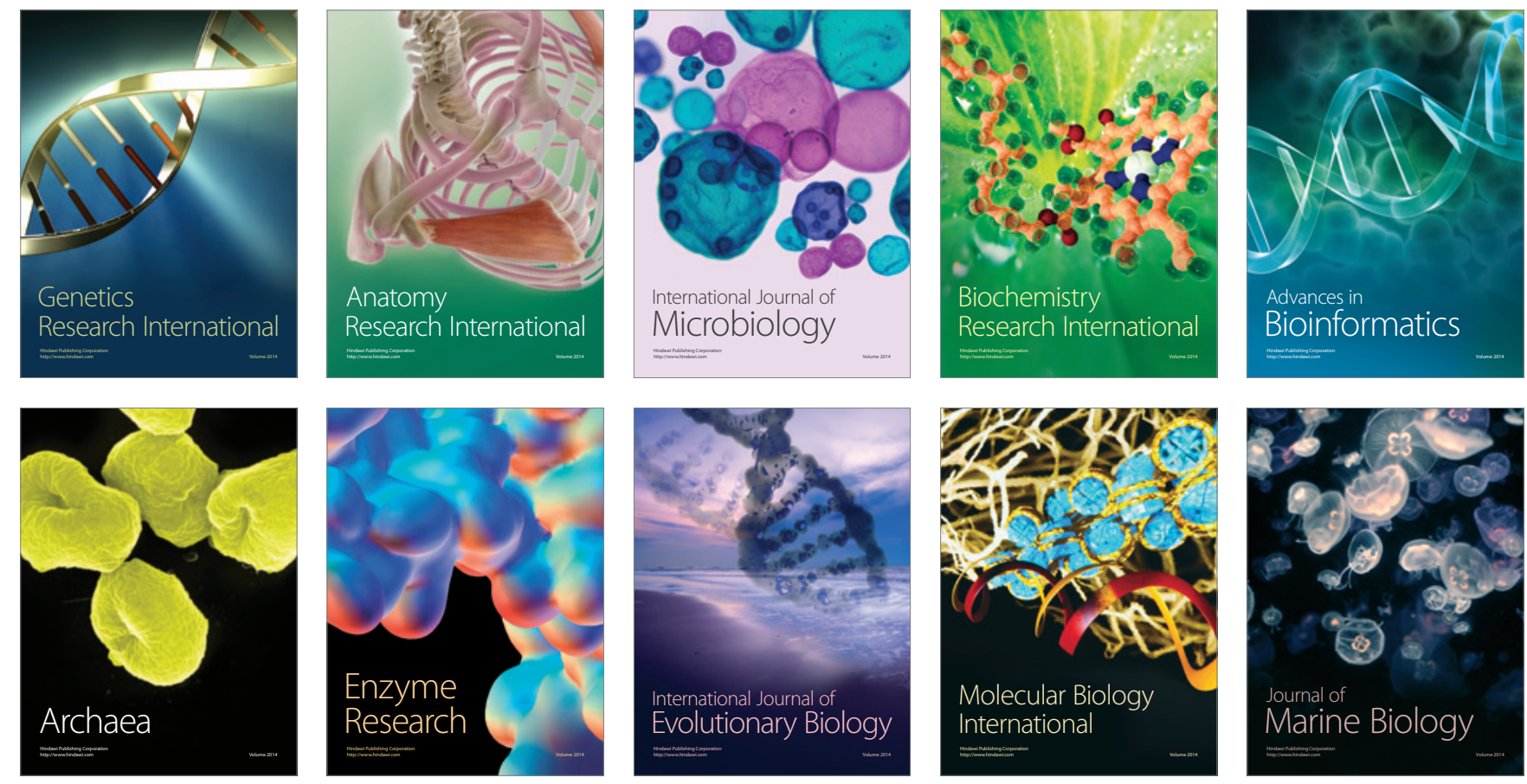